\title{
The Comparative Study of Chinese and English Kinship Terms
}

\author{
Yan Miao \\ Shanxi Normal University, China
}

\begin{abstract}
Kinship terms were an important part in communication, existing in all countries in the world. With the development of cultural exchange, the comparison and analysis between Chinese and English kinship terms becomes more vital and necessary. The author will put forward some suggestions about Chinese kinship terms teaching for international students based on comparison and analysis between the two language systems. This paper will be divided into five parts. Firstly, the author will introduce the concept, content, present situation, research methods and significance of kinship terms. Secondly, the author will pay attention to point out their features and classification. Thirdly, the author will describe their similarities and differences. Fourthly, the author will put forward some suggestions about Chinese teaching for foreigners. Finally, the author will make a summary and point out the limitation in the paper.
\end{abstract}

Index Terms — kinship terms, culture, Chinese teaching for international students

\section{INTRODUCTION}

As a common language phenomenon, kinship terms were used in daily communication. Researching and comparing diverse cultural kinship terms are a necessary method to learn and understand different national history and culture. China and Western countries communicate frequently, a comparative study of kinship terms is becoming more and more significant. This author will focus on the researches of kinship terms and find out some good teaching methods for International students.

This paper will be classified into five parts to study kinship terms. To begin with, the author will introduce the concept, content, present situation, research methods and significance of modern kinship terms. Secondly, the author will pay attention to the researches of Chinese and English kinship terms, and point out their features and introduces their classification. Thirdly, the author will analyze their similarities and differences. Fourthly, the author will put forward some suggestions of Chinese teaching for foreigners. Finally, the author will summarize the significance, the limitation and difficulties in this paper.

\section{LITERATURE REVIEW}

Researches to kinship terms are numerous and multiple, but the learners have been not an agreement on the definition of the kinship terms, yet. There were three viewpoints in present situation. First opinion: kinship terms were divided into generalized and narrow kinship terms. Second opinion: there was no difference in the kinship terms and salutations. Third opinion: salutations were a part of the kinship terms. The three views above all are advisable opinion, as far as the author is concerned, Mr. Cui's definition about kinship terms was more convinced and reliable. He (1996) thought that the kinship terms not only represent one's social relationship, but also the identity in society; the salutations were just used in the occasion where people communicate face to face (Cui, 1996). Based on the descriptions above, the definition of the kinship terms is people's appellations used in mutual communication and relationship for relatives' relationship or social identities and occupations.

After researching the recent studies about modern Chinese kinship terms, the contents of present situation can be classified roughly into three parts. The first part focused on the method of comparison and analysis of the modern Chinese kinship terms itself. For example, Li Shuxin (1990) had discussed the Chinese kinship terms from the cultural aspect in Modern Chinese Kinship Terms and Traditional Culture (Li, 1990). The second part focused on the comparison and analysis between Chinese and other languages' kinship terms, which accounts a largest portion in the researches the author collected. In The Differences and Cultural Factors between Chinese and English Kinship Terms, Chen Lingmei (2009) said: "Chinese and English kinship terms are different"(Chen, 2009, p.143). The third part focused on the researches about cultural translation based on the Chinese kinship terms; this kind of research aims to find out the methods how to translate Chinese kinship terms in an effective way, and make the foreign readers understand Chinese traditional culture in an original way and reaches the point of culture transmission. The final part focused on Chinese kinship terms teaching for foreigners. Cui Xiliang (1996) had putted forward some suggestions and measures about Chinese teaching for foreigners in Modern Chinese kinship Terms and Chinese-teaching to foreigners (Cui, 1996). In a word, many learners have studied and analyzed Chinese kinship terms in detail, making the modern Chinese kinship terms studies reach a mature stage. 
Foreign learners had combined kinship terms with linguistics, sociology, psychology, culture, anthropology and ethnology. Social linguistics, as a discipline, sprung up in the 1960s in the Europe and America. The language and communication in social life were the main object of social linguistics; and its contents varied from macrocosm to microcosm, creating scientific and objective researches. Since Roger Brown and Albert Gilman (1960) disscussed the kinship terms in The Pronouns of Power and Solidarity (Brown \& Gilman, 1960), the studies about kinship terms have been carried out widely, forming rich and fruitful achievements. Marguerite Ford published Address in American English in 1964 and researched deeply the usage patterns of American kinship terms in the method of questionnaire and documentary research. H. W. Scheffiler (1985) adopted the form of diagrams to study English kinship terms in Theory and method in the study of kinship (Scheffiler, 1985), which was almost unprecedented in the processor of researching the kinship terms.

The studies about kinship terms are rich and fruitful, and a lot of phonographs have been published since 1979. After inputting the key words-kinship terms in CNKI, the author can find 641 published articles in many periodicals and magazines since 1985 and 214 master's paper based on the kinship terms since 2000. But those articles and magazines paid more attention to the comparison between Chinese and foreign languages kinship terms, which mainly focuses on general description, rarely on specialized and detailed studies of kinship terms. There are few studies to research and study the kinship terms from the aspect of Chinese-teaching. Based on descriptions above, the studies about modern kinship terms are necessary and essential under the situation of frequent communication between different countries.

The paper contains three important parts. The first part focus on describing objectively the classification, characters and cultural connotation of Chinese and English kinship terms through collecting and summarizing former researches. The second part paid attention to the comparison of kinship terms between Mandarin in China and English in western countries; and found out the reasons caused differences between them. The third part focused on the way to introduce the cultural words for foreign students effectively, making them understand traditional Chinese culture deeply.

\section{The Systems OF CHINESE AND ENGLISH KINSHIP TERMS}

Vinous kinship terms have leaded to a more complex system about kinship terms in different countries, reflecting relationships among people in different degrees. When researching books and magazines, the author found that kinship terms can be classified into many parts from different angles and there were lots of classification methods. Compared Chinese kinship terms, the classified methods about English kinship terms are ambiguous and indistinct. The classification methods are different under the different culture background, Chinese kinship terms belong to general classification method, English kinship terms belong to narrative classification method. The author paid attention to kinship terms within four generations due to the complexity of kinship terms.

\section{A. The Summary of Chinese Kinship Terms}

The system of Chinese kinship terms is one of the most detailed systems, and experts have not reached an agreement on it, urging us to learn and understand it for benefiting to Chinese foreign teaching.

1. The classification of Chinese kinship terms

According to books and magazines, the author learned that there were many classification methods. In Chinese Culture and Translation between English and Chinese, Lu Hongmei (2006) said: "Chinese kinship terms could be classified into aspectant appellation and indistinct appellation" (Lu, 2006, p. 31). For example 'mommy' belongs to former and 'mother' belongs to later. In addition, the two-classified method, four-classified method and ten-classified method all were very common and familiar for us. Hu Shiyun (2007) adopted the ten-classified method to classify Chinese kinship terms in The Research of Chinese kinship terms (Lu, 2007). So the author concludes that Chinese kinship terms are complicated, experts and scholars have not come to agreement on some proper nouns of Chinese kinship terms, making foreign learners learn and understand difficultly. For carrying out works of Chinese teaching for international students, the author will introduce clearly Chinese kinship terms in this paper.

Firstly, the author will analyze some proper nouns. According to the reasons of formation, kinship terms were classified into spouse, consanguinity and in-laws relationship. Consanguinity relationship contains direct descent and indirect descent. The first refers to the relationships of birth and rearing; consanguinity refers to the indirect relative blood relationship; In-laws relationship is based on the spouse relationships. Spouse refers to the couples with legal marriage relationship, which is the foundation of other relationship.

Secondly, clan relatives refer to the natural kinship based on the blood relationship, and contain maternal line and paternal line in ancient times. The former refers to the relationship among people who have common ancestor. The latter refers to the relationship produced in a condition where one marrys with women. So the author concludes that the two-classified method is based on the research about traditional Chinese kinship terms. The four-classified method and ten-classified method were based on modern restrictive. Combined previous experience with Chinese teaching as a foreign language, the author tries to look for a classification method benefiting to Chinese teaching for foreigners. So, the author will combine Cui Xiliang' s classification method with reasonable parts of the two-classified method and four-classified method, dividing Chinese kinship terms into three parts.

2. The features of Chinese kinship terms

The systems of kinship terms are plentiful and different in different cultural background. Chinese kinship terms cover 
many aspects of language and there are many classification methods.

Gender is a important and clear line between men and women. The differences between men and women not only reflect differences of physiological feature, but also the difference of social aspects. The physiological difference between them is a symbol distinguished kinship among people in the system of Chinese kinship terms. Chinese kinship terms combine gender, attribution with people's family name. Thanks to different gender of parents, the difference of appellation between matriarchal and patriarchy produced naturally. For example, the word 'aunt' in Chinese belongs to patriarchy; her family name is same as her father and different with her mother and children.

Chinese focus on the morals view of respecting the older in Chinese kinship terms. The view of 'nine' clans occurred in China ancient times and reflected in Chinese kinship terms. Since the Western Zhou, Chinese had cherished blood relationship very much. After the foundation of the People's Republic of China, Chinese have weakened the consciousness of 'nine clans', but the number of significant and crucial Chinese kinship terms is still more than twenty even in the modern China.

Chinese kinship terms focus on distinguishing the differences between patriarchal and maternal. For example, grandfather (patriarchal), grandfather (maternal) both have same gender and position in a family hierarchy, because they belong to different factions of kinship, their appellations in Chinese kinship terms are different.

Differences among clans are clear. The view of clans was related to one's family name. For example, uncle (patriarchal) belongs to patriarchal relatives, uncle (maternal) belongs to maternal relatives. If one person and the children of his patriarchal relatives both have the same family name, they belong to the same clan. But this person's family name is different with the children of his maternal relatives, which reflects this person and the children of his maternal relatives do not belong to the same clan.

\section{B. The Summary of English Kinship Terms}

After learning Chinese kinship terms, the author will introduce the classification and features of English kinship terms.

1. The classification of English kinship terms

The author learned that Englishman prefer to call their relatives' name directly rather than appellations in daily communication. The author concludes that English kinship terms belong to direct system and classifies English kinship terms into core and general kinship terms from the aspect of core family (a family contains father, mother and unmarried children) and general family. Core kinship terms refer to names belong to the core family, such as, father, mother, husband, and wife. General kinship terms refer to names belong to general family, such as, grandfather, grandmother, uncle.

\section{The features of English kinship terms}

Based on the previous researches, the author summarized features of English kinship terms below.

In spoken English, Westerners do not use appellations to call others, such as, sister and brother. English kinship terms can be classified into official appellations and intimate appellations in different occasion.

English kinship terms are generalized. For example, people cannot distinguish one's gender and compare two person's age from the English word 'cousin'.

The appellations of direct relation and collateral relation are different in same generation. For example, Englishman call their children 'son' or 'daughter', they call the children of their sister or brother 'nephew' or 'niece'.

The Gender discrimination has existed in Western countries long time, such as, Mrs. / Miss and Mr., Ms. and Mrs. are the call of women. But Miss is the name of unmarried women, Mr. is the name referred to all men, which can not reveal intimate information about man. The way of using appellations caused inequality between men and women. With the development of feminist movement, 'Ms' refers to all women, occurring in modern society.

Englishman prefers to call people's name rather than appellations. Even the phenomena that children call his mother's name in movies are common and natural.

\section{The COMPARISON AND ANALYSIS OF CHINESE AND ENGLISH KINSHIP TERMS}

After learning Chinese and English kinship terms, the author will compare and analyze the two systems for carrying out works of Chinese teaching.

\section{A. The Similarities between Chinese and English Kinship Terms}

The two systems both reflect the relationship among the members of core family, such as, father, mother and unmarried children.

The two systems both contain appellations of blood relationship, such as, the word 'uncle'.

The two systems both contain appellations reflected the relationship among two person who are related with each other because of marriage, such as, mother-in-law, and father-in-law.

The two systems both can distinguish person's gender and age. For example, the author can judge one's' gender from the word 'uncle' in English. (The word 'cousin' is a special word and contains two genders.)

B. The Differences between Chinese and English Kinship Terms 
Based on the descriptions above, Chinese and English kinship terms have many similarities, but the differences of the two systems are more than their similarities. And, the author will analyze the differences between Chinese and English kinship terms.

The aspect of distinguishing one's gender is different. One's gender can be distinguished clearly from the Chinese kinship terms, but English cannot be. For example, the word 'cousin' can not only represents a man, but also a woman in English.

Chinese and English kinship terms cannot be translated absolutely to each other. The scope of English kinship terms is larger than Chinese, and the former are more generalized than the latter. For example, the word 'grandfather' in English can not only represent the father of one's father, but also can represent the father of one's mother in English.

The degree of extension and generation of the two appellation system is different. Chinese kinship terms are more common than English kinship terms in daily communication. For example, Chinese prefer to call a strange old man 'grandfather'. But Englishman prefers to say 'Hello' or 'Excuse me' to communicate with stranger in first communication.

\section{The Reasons of Cultural Differences between Chinese and English Kinship Terms}

Based on the description above, the author will introduce and analyze the reasons caused the cultural difference between the two systems.

Since the ancient time, Chinese have attached importance to the view of family and blood relationships. Traditional views of clans have influenced the usage of Chinese kinship terms deeply. Based on natural economy, feudal society had existed in China for thousands years, making Chinese pay more attention on blood relationships; The families composed of four generations are natural and common in China. So, Chinese kinship terms are vital and necessary to Chinese very much. But, westerners paid more attention to freedom and independence due to the Industrial Revolution. They like little and core family rather than big family of four generations, attaching little importance to blood relationship than Chinese. So, the system of Chinese kinship terms is detailed, the system of English kinship terms is generalized.

Chinese have attached importance to collectivism, and collective interests are more important than personal interests in China. Chinese always subordinate personal interests to collective interests, causing that Chinese are accustomed to extending the scope of using of appellations to the social relationship. But the Westerners want to pursuit the freedom, equality and democracy. As the Declaration of Independence mentioned,

We hold these truths to be self-evident, that all men are created equal, that they are endowed by their Creator with certain unalienable rights, that they are among these are life, liberty and the pursuit of happiness.

Westerners prefer to call one's name directly in daily communication.

As to the differences of national character between China and Western countries, Chinese are moderate in temper, they focus on harmony, blood relationships and public interests and always prefer to extend the scope of using the kinship terms in society to create a harmonious atmosphere. The people of Western countries focus on the pursuits of freedom, equality, democracy and the realization personal value. They tend to create a kind of impartial and equal atmosphere. So, Westerners are more aggressive than Chinese.

Different concepts of child-bearing and the number of children in a family is a key factor caused the cultural difference between China and Western countries. Chinese like big family composed of some generations. There were lots of words and phrases to describe big family in Chinese dictionaries, for example, 'There is strength in numbers,' 'with luxuriant foliage,' 'Flourishing population'. The big family composed of some generations is the foundation of the whole society in China. So the kinship terms are necessary for Chinese to distinguish blood relationships among people. But the core family is more popular in Western countries after Industrial Revolution. The children will leave their parents and make a living for themselves after growing up, which leads to English kinship terms are more generalized and simple.

\section{The Elicitation of Chinese TeACHING FOR Foreigners THROUgh COMPARISON AND ANALYSIS OF THE Two} SYSTEMS

Based on the description above, the author thinks Chinese kinship terms teaching is important, and will put forward some suggestions about it.

\section{A. The Importance of Chinese Kinship Terms Teaching}

Kinship terms are important in daily communication and decent kinship terms promote to communicate with other people successfully. But Chinese kinship terms are difficult to learn and understand for international students, especially English students and American students. Because the Chinese kinship terms are detailed and there were many ways of classification. Foreigners always are confused by the same kind of questions, for example, why Chinese call a strange old woman grandmother. With different cultural background, foreign learners are not familiar with the cultural connotation of Chinese kinship terms, they hold a view that Chinese kinship terms are difficult for them to learn and understand. In foreign learners' view, the Chinese kinship terms are complex and various, the English kinship terms are generalized and simple. If scholars do not compare and analyze difference and reasons caused the differences between 
the two systems, foreign learners are more likely to learn the Chinese kinship terms in the rule of learning English, which will lead to the phenomenon of 'negative transfer' and obstacles of communication. So the Chinese kinship terms teaching are necessary to research.

\section{B. The Suggestions of Chinese Kinship Terms Teaching}

Due to complex kinship terms in Chinese, a lot of foreign learners cannot understand truly its meaning and usage. Combined the questions existed in Chinese teaching with the comparison and analysis between the two systems, the author will put forward some suggestions about Chinese teaching for foreign learners.

1. The suggestions about selecting textbook

When selecting textbook, the workers should pay attention to pertinent, practical, interesting, scientific and systematic principle. For example, the introduction of the Chinese kinship addressed in textbook is not systematic, generalized and comprehensive, the workers should combine Western culture with the real life in China when selecting textbooks.

2. The suggestions about teaching in class

As a special group, teachers play an important role during the process of spreading information. So, teachers should possess comprehensive teaching ability to select textbook, create suitable learning situation for students, arose students' learning enthusiasm and improve promote teaching quality.

Firstly, students must have a command of core kinship terms, such as, mother, father, sister, brother. Teachers should help students memorize and practice Chinese kinship terms.

Secondly, students must have a command of general kinship terms, such as, uncle, aunt. Teachers should combine cultural background of China and Western countries with traditional view to teach kinship terms, making international student have a comprehensive command of Chinese kinship terms.

Finally, teachers should not only introduce the usage and features of Chinese kinship terms to students, but also analyze the hidden values and mentality of kinship terms. More importantly, teachers should create various situations for students, making students use it familiarly and naturally.

3. The suggestions about culture teaching

With the significant difference between the two systems, teachers should adopt various teaching methods to cultivate students' sensitivity and help them understand deeply cultural phenomenon. The author will put forward some teaching methods with the combination of previous researches and practical experience of learning second language, such as, cultural short drama, discussion about culture.

\section{CONCLUSION}

The author combined previous research with the present situation, and came to three conclusions:

Firstly, the author gave a definition of the kinship terms, it refers to the special identity, position in the clans, one's gender, and represent relationships reflected in whole family or society.

Secondly, the author classified Chinese kinship terms into terms of direct and indirect blood relationship and introduced their features. Then the author classified English kinship terms into core and general kinship terms and introduced their features.

Thirdly, the author found that their differences are more than similarities. Trough analyzing the cultural differences between them, the author concluded that the structure of family and society, traditional view, national character and the view of child-bearing all lead to the difference between Chinese and English kinship terms.

Finally, the author putted forward some suggestions of Chinese teaching. For example, cultural short drama, discussion about culture, the comparison between ancient times and modern society.

This paper still has some shortcomings. Even though, the author hopes that it will provide practical information about Chinese learning for international students.

\section{REFERENCES}

[1] Cheng Lingmei. (2009). The Differences and Cultural Factors between Chinese and English Kinship Terms. China Electric Power Education, 1, 142-144.

[2] Cui Xiliang. (1996). Modern Chinese kinship Terms and Chinese-teaching to foreigners. Language Education and Research, 2 , 34-47.

[3] Hu ShiYun. (2007). The Research of Chinese kinship terms. Beijing: The Commercial Press.

[4] H. W. Scheffiler. (1985). Theory and method in the study of kinship. Review Anthropology, 12(1). 12-17.

[5] Lu Hongmei. (2012). Chinese Culture and Translation between English and Chinese. Wuhan: Wuhan University Press.

[6] Li Shuxin. (1990). Modern Chinese Kinship Terms and Traditional Culture. Journal of Inner Mongolia University, 3, 54-59.

[7] Roger Brown, \& Albert Gilman. (1960). The Pronouns of Power and Solidarity. Columbus, OH: Charles E. Merril Press.

[8] Sunstein Cass R. (2003). The Declaration of Independence. Washington: Georgetown University Press.

[9] Saad Shekish. (2009). Combinatorial Methods in Kinship Analysis. Illinois: University Illinois at Chicago. 
Yan Miao was born in Shanxi, China in 1990. She graduated from North University of China in 2001. She is currently a postgraduate in Shanxi Normal University, China. Her major is English Translation. 